\title{
Quantitative hemodynamic studies in moyamoya disease
}

\author{
A review \\ Marco Lee, M.D., Ph.D., F.R.C.S., ${ }^{1}$ Greg Zaharchuk, M.D., Ph.D., ${ }^{2}$ \\ Raphael GuZman, M.D., ${ }^{1}$ Achal Achrol, M.S., ${ }^{1}$ Teresa Bell-Stephens, R.N., ${ }^{1}$ \\ and Gary K. Steinberg, M.D., Ph.D. ${ }^{1}$ \\ ${ }^{1}$ Department of Neurosurgery, Stanford Stroke Center; and ${ }^{2}$ Department of Radiology, Stanford University, \\ Stanford, California
}

\begin{abstract}
Moyamoya disease is characterized by a chronic stenoocclusive vasculopathy affecting the terminal internal carotid arteries. The clinical presentation and outcome of moyamoya disease remain varied based on angiographic studies alone, and much work has been done to study cerebral hemodynamics in this group of patients. The ability to measure cerebral blood flow (CBF) accurately continues to improve with time, and with it a better understanding of the pathophysiological mechanisms in patients with moyamoya disease. The main imaging techniques used to evaluate cerebral hemodynamics include PET, SPECT, xenon-enhanced CT, dynamic perfusion CT, MR imaging with dynamic susceptibility contrast and with arterial spin labeling, and Doppler ultrasonography. More invasive techniques include intraoperative ultrasonography. The authors review the current knowledge of CBF in this group of patients and the role each main quantitative method has played in evaluating them, both in the disease state and after surgical intervention. (DOI: 10.3171.2009.1.FOCUS08300)
\end{abstract}

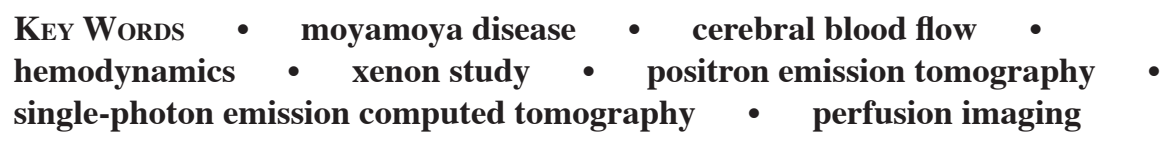

$\mathrm{S}$ INCE Suzuki and Takaku's ${ }^{31}$ landmark paper in 1969, in which the term "moyamoya disease" was coined, our understanding of the clinical manifestation and natural history of the disease has grown. However, it remains a disease of unknown origin. It is characterized angiographically by the progressive stenosis or occlusion of the terminal ICAs. This is often accompanied by the development of collateral networks, which seemingly provides an alternative route for cerebral perfusion. However, this pathophysiological bypass is imperfect. The attempt to compensate for reduced cerebral perfusion often fails, leading to the well-known clinical manifestations of the disease, including ischemic strokes and

\footnotetext{
Abbreviations used in this paper: $\mathrm{ASL}=$ arterial spin labeling; $\mathrm{CBF}=$ cerebral blood flow; $\mathrm{CBV}=$ cerebral blood volume; $\mathrm{CMRO}_{2}$ $=$ cerebral metabolic rate of oxygen; $\mathrm{CPP}=$ cerebral perfusion pressure; CVR = cerebrovascular reserve; $\mathrm{DSC}=$ dynamic susceptibility contrast; ICA = internal carotid artery; MTT = mean transit time; $\mathrm{OEF}=\mathrm{O}_{2}$ extraction fraction; $\mathrm{rCBF}=$ regional $\mathrm{CBF} ; \mathrm{rOEF}$ = regional OEF; STA-MCA = superficial temporal artery-middle cerebral artery; TIA $=$ transient ischemic attack; Tmax $=$ normalized time to the bolus peak; Xe-CT $=$ xenon-enhanced CT.
}

TIAs. Moreover, the fragile collateral networks have a propensity to bleed, which may result in hemorrhagic strokes.

Examination of the angiographic studies can give some insight into the severity of the disease. However, there is often a disparity between the angiographic picture and the clinical severity of the disease. Patients may have bilateral occluded ICAs with florid collateral vessels and remain asymptomatic. In contrast, patients with angiographically confirmed mildly stenotic or preocclusive disease may experience severe ischemic symptoms. This most likely reflects the complex interplay of the many factors that govern CBF, including age, systemic factors, the quality of the rest of the cerebral circulation, and the collateral networks. Furthermore, there are intrinsic limitations of angiographic studies used to evaluate CBF in a qualitative and quantitative manner.

It is worth noting that just over 50 years ago, most strokes were thought to be due to vasospasm. ${ }^{4}$ Thromboembolic disease is now recognized as the leading cause of stroke, and the pathological process of clot formation and dissociation is well understood. However, our knowl- 
TABLE 1: Imaging techniques used to evaluate moyamoya disease*

\begin{tabular}{|c|c|c|c|c|c|c|}
\hline Modality & Contrast & $\begin{array}{l}\text { Ra- } \\
\text { diation } \\
\text { Dose } \\
\text { (mSv) }\end{array}$ & $\begin{array}{l}\text { Hemodynamic } \\
\text { Measures }\end{array}$ & $\begin{array}{c}\text { Brain } \\
\text { Coverage }\end{array}$ & Quantitative Accuracy & $\begin{array}{c}\text { Vari- } \\
\text { ability } \\
(\%)\end{array}$ \\
\hline PET & ${ }^{15} \mathrm{O}_{2}, \mathrm{C}^{15} \mathrm{O}_{2}, \mathrm{H}_{2}{ }^{15} \mathrm{O}$ & $0.5-2$ & CBF, CBV, rOEF & whole & good & 5 \\
\hline SPECT & $\begin{array}{l}{ }^{133} \mathrm{Xe},{ }^{99 m} \mathrm{TC}-\mathrm{HMPAO}, \\
{ }^{99 \mathrm{~m} T c-E C D},{ }^{123} \mid-\mathrm{IMP}\end{array}$ & $3.5-12$ & $\mathrm{CBF}$ & whole & good for ${ }^{133} \mathrm{Xe},{ }^{123}|-| \mathrm{MP}$ & 10 \\
\hline $\mathrm{Xe}-\mathrm{CT}$ & stable xenon gas & $3.5-10$ & CBF & $6 \mathrm{~cm}$ thick & good & 12 \\
\hline $\begin{array}{l}\text { dynamic } \\
\text { perfusion CT }\end{array}$ & iodinated & $2-3$ & CBF, CBV, MTT, Tmax & $4-5 \mathrm{~cm}$ thick & moderate & $10-15$ \\
\hline DSC MRI & $\mathrm{Gd}$ & none & CBF, CBV, MTT, Tmax & whole & - & $10-15$ \\
\hline ASL MRI & none & none & $\mathrm{CBF}$ & whole & moderate & 10 \\
\hline Doppler US & none & none & ICA-BFV & hemisphere & good for hemispheric CBF & 5 \\
\hline
\end{tabular}

edge of ischemic stroke caused by hemodynamic insufficiency, such as that encountered in moyamoya disease, has lagged behind. This has mostly been due to the difficulty of quantifying $\mathrm{CBF}$ and interpreting the results. Over the years, numerous quantitative techniques for $\mathrm{CBF}$ and other hemodynamic parameters have evolved. Patients with moyamoya disease have played a particularly important role in helping us understand the advantages and disadvantages of each technique, and the interpretation of the data have similarly brought greater understanding of this challenging disease.

\section{Quantitative Methods of Measuring Cerebral Hemodynamics}

An ideal method to quantify cerebral hemodynamics would involve a noninvasive technique that is instantaneous and repeatable. It would also generate data that accurately reflect regional and total CBF. In 1948, Kety and $\mathrm{Schmidt}^{10}$ developed a quantitative method to measure whole-brain $\mathrm{CBF}$ and the $\mathrm{CMRO}_{2}$ in humans, based on the Fick principle. Their method required arterial and

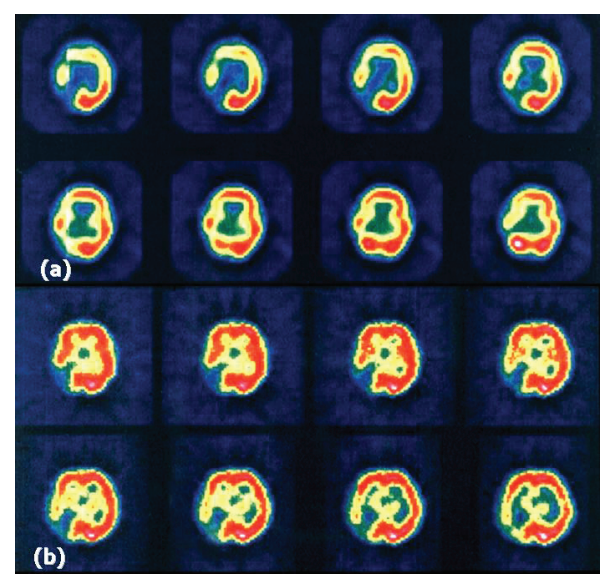

FIG. 1. Hemodynamic findings on SPECT studies obtained pre- (a) and postacetazolamide (b) in a 34-year-old patient with bilateral moyamoya disease and previous right occipital artery stroke. internal jugular blood sampling while the patient inhaled nitrous oxide. Other similarly invasive techniques were developed to measure $\mathrm{rCBF}$, and our knowledge of normal $\mathrm{CBF}$ values from these classic experiments has stood the test of time. Modern imaging modalities have allowed much less invasive techniques, but the measurements derived from PET, SPECT, and Xe-CT are still based on the Kety-Schmidt model. These techniques have been widely used to evaluate patients with moyamoya disease. With the advent of MR imaging, other techniques, including DSC and ASL, have also been applied to cerebral hemodynamic analysis in moyamoya disease. ${ }^{37}$ Table 1 summarizes the main imaging techniques applied to the study of moyamoya disease.

A detailed discussion of the various quantitative hemodynamic techniques is beyond the scope of this paper, and the reader is referred to a recent review. ${ }^{40}$ Nevertheless, it is not possible to understand fully the cerebral hemodynamics literature in moyamoya disease without an appreciation of the strengths and limitations of each technique.

Oxygen-15 PET Analysis. This modality has been

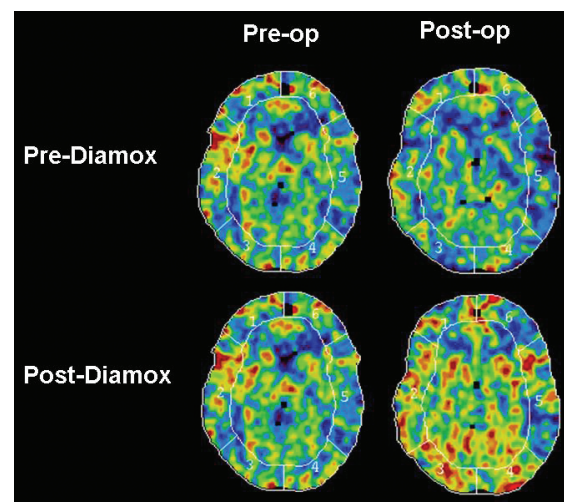

FIG. 2. Preoperative and 1-year postoperative Xe-CT studies obtained with and without acetazolamide (Diamox) in a 45-year-old patient with bilateral moyamoya disease, which was worse in the left hemisphere. 


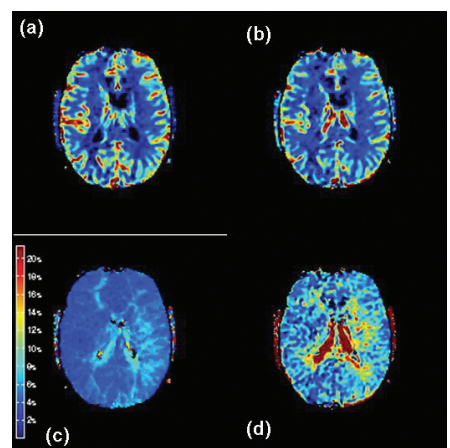

FIG. 3. Hemodynamic findings on MR perfusion studies obtained in a 20-year-old patient with bilateral moyamoya disease, with prolonged Tmax and MTT bilaterally, but worse in the left hemisphere. These MR perfusion studies demonstrate CBF (a); CBV (b); Tmax (c); and MTT (d).

widely used to study patients with moyamoya disease. ${ }^{6,8,21,22}$ It enables multiple hemodynamic parameters to be measured. In addition to $\mathrm{rCBF}$ and $\mathrm{CMRO}_{2}$, regional $\mathrm{CBV}$ and $\mathrm{rOEF}$ (which represents the fraction of $\mathrm{O}_{2}$ consumed by the brain) can also be measured. The significance of these parameters is discussed below. The main disadvantage of PET imaging is that it is much less available and relatively more costly. The PET tracers require the availability of a cyclotron to produce the very short-lived radiopharmaceutical material (half-life 2 minutes).

The SPECT Modality. This method is much more readily available. However, it is not strictly quantitative, and the rCBF map it generates is statistically compared with a normal control to represent regions of abnormal perfusion. It also has a poor spatial resolution. Despite these limitations, it has been frequently used in studies of moyamoya disease and in postsurgical CBF evaluations..$^{27,30,38}$ It is often used in conjunction with a vasodilatory challenge (for example, acetazolamide challenge) as an assessment of functional reserve, as will be discussed below (Fig. 1).

Xenon-Enhanced CT Studies. This modality has been a workhorse of CBF measurements for $>20$ years. Again, it has been widely used to study moyamoya disease, and in conjunction with the acetazolamide challenge, it is frequently used to determine the suitability of revascularization surgery and to evaluate the postoperative CBF. $5,18,20,32$ Its main disadvantage is that it has a relatively long acquisition time, it is prone to motion artifacts, and some patients cannot tolerate inhalation of xenon through a face mask during the procedure. Furthermore, due to regulatory issues, the $\mathrm{Xe}-\mathrm{CT} \mathrm{CBF}$ technique is not readily available at present in many countries, including the US (Fig. 2).

Perfusion CT Scanning. This is a relatively new technique that measures CBF by using a first-pass tracer methodology. It has gained popularity as the availability of helical CT scanners capable of operating in the cine mode has grown, and there have been a few reports of this technique being used in moyamoya research. ${ }^{7,26,28}$ The CBF, CBV, and MTT maps can be easily generated.

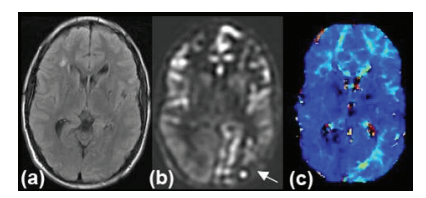

FIG. 4. Neuroimages obtained in a 19-year-old man with moyamoya disease, including FLAIR (a); ASL (b); and bolus DSC (c) MR imaging (Tmax). This patient has involvement of his left posterior cerebral artery territory (arrow), which is clearly distinguished from the normal perfusion in the right posterior cerebral artery territory. The prolonged blood arrival time is confirmed on perfusion-weighted imaging Tmax maps.

Quantitative CBF measurement requires the masking of voxels containing large arteries and veins. ${ }^{39}$ It also presents problems involving delay and dispersion of the contrast bolus (similar to MR imaging; see below), in which flow can be underestimated. The great advantages of the technique include the large base of CT scanners installed throughout the world, the speed of the examination, and the lack of significant contraindications. Disadvantages include the radiation dose and the fact that iodinated contrast material may be unsuitable for some patients.

Newer MR Imaging-Based Methods. These methods, such as DSC-weighted bolus-tracking MR imaging ${ }^{37}$ and ASL, are the latest additions to the growing number of techniques available for quantitative hemodynamic analyses. The DSC imaging modality also relies on firstpass tracer methodology. After a bolus dose of contrast is given, ultrafast images are acquired and various hemodynamic parameters are calculated using commercially available software. The most common parameters generated include Tmax, apparent MTT, CBV, and CBF index (calculated as CBV/apparent MTT). Most recent approaches have used a deconvolution of the arterial input function with the tissue tracer curve to improve quantitation and limit the effects of bolus delay ${ }^{24}$ (Fig. 3).

The ASL MR imaging modality requires no contrast media because it uses endogenous water as a tracer. ${ }^{3,15}$ Water in the blood is magnetically labeled upstream of the brain within the cervical arteries. Then, images are acquired to assess the subsequent distribution of this water label, which enters the brain parenchyma in proportion to local CBF. Images with and without the spin label are acquired and then subtracted, leading to problems if there is significant patient motion. This method is becoming more clinically relevant, given recent improvements in pulse sequences, which have reduced artifacts and increased the sensitivity of the examination. Additionally, the method is significantly improved by the use of high-field (that is, $\geq 3-\mathrm{T}$ ) MR imaging units. Advantages include lack of radiation, lack of intravenous contrast, the ability to repeat the study, and the ability to compare $\mathrm{CBF}$ values within and between patient examinations. The main disadvantage in using this technique to study moyamoya disease is that the delayed arterial times through collateral pathways can cause a significant underestimation of $\mathrm{CBF}$ as well as errors due to labeling agent that remains in the feeding vessels rather than being extracted into the brain parenchyma. All MR imaging-based techniques are of course associated with a substantial number of patients who are either unsuitable or cannot tolerate lying inside a magnet (Fig. 4). 


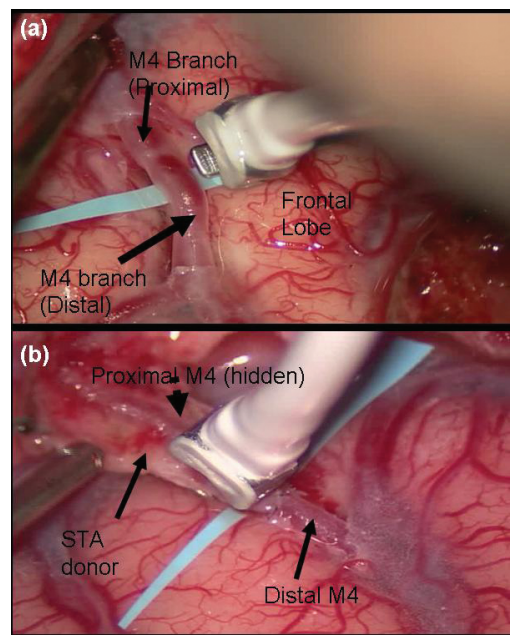

FIG. 5. Intraoperative photographs showing blood flow monitoring with the Charbel Transonic Flowprobe before (a) and after (b) STA-MCA microanastomosis for moyamoya disease.

Doppler Ultrasonography. This modality has been used in many moyamoya studies. ${ }^{16,17,19}$ It is a noninvasive technique that is quite available and can be performed at the bedside. However, it is very operator dependent and prone to spurious results. It can be used to measure blood flow volume in the ICA as a correlate for $\mathrm{CBF}$ in the corresponding hemisphere. However, this hemispheric CBF is an estimation based on the ICA blood flow volume measurement. It has poor spatial resolution because it cannot relay information on focal CBF impairments.

Flowprobes. All the techniques so far discussed involve indirect measurements of rCBF. However, during revascularization surgery, especially for moyamoya disease, direct measurement of blood flow locally is feasible. A flexible perivascular flowprobe (Charbel; Transonic Systems, Inc.) can be positioned over a target vessel that has been dissected free of the surrounding tissue. The flowmeter uses an ultrasonic transit-time principle to measure volumetric flow directly in milliliters/minute. We have used this technique extensively to measure local blood flow in patients with moyamoya disease and to correlate the results with those obtained from the $\mathrm{Xe}-\mathrm{CT}$ and SPECT studies (Fig. 5).

Quantitative MR Angiography. More recently, the development of quantitative MR angiography has also allowed the local blood flow rate across a specific vessel to be measured noninvasively. We have begun to apply quantitative MR angiography in the form of a commercially available software program (NOVA; VasSol, Inc.) to evaluate patients with moyamoya disease before and after revascularization procedures. Comparison of blood flow rates and direction can be made with data obtained intraoperatively, and the changes in the flow rate and direction after microanastomosis can be studied with time. It remains to be seen if this technique will be clinically useful in evaluating patients or guiding surgical decisions (Fig. 6).

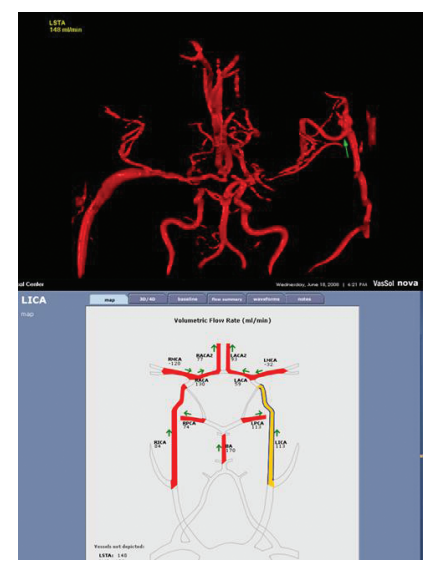

FIG. 6. Studies performed using the NOVA software to assess results in a 55-year-old patient with bilateral moyamoya disease 1 year after STA-MCA bypass on the left side. Blood flow is measured on various arteries of the circle of Willis and the left STA-MCA graft (arrow). Note that a previous right saphenous vein graft can also be seen and measured.

\section{Pathophysiological Mechanisms Affecting CBF}

Normal CBF values have been reported to be $\sim 45-$ $55 \mathrm{ml} / 100 \mathrm{~g} / \mathrm{min}$, and flow is affected by alterations of the CPP and vascular resistance. Through autoregulation, there is a reflex vasodilation of resistance arterioles, and CBF remains constant even when CPP changes within the range of 50-150 $\mathrm{mm} \mathrm{Hg}$. Stenosis or occlusion of a cerebral vessel may cause CPP reductions beyond the autoregulatory capacity, resulting in a fall in the CBF. This is accompanied by an increase in OEF, which will maintain $\mathrm{CMRO}_{2}$ and tissue function temporarily (so-called misery perfusion). However, further reductions of CPP will exhaust the OEF compensatory response and lead to irreversible ischemia. The reflex vasodilatory response is also thought to explain the increase in CBV observed with falling CPP. However, there are conflicting results that also show that CBV may only increase significantly when CPP falls beyond the autoregulatory range., ${ }^{2,41}$

The CBV is an important concept to consider when evaluating patients with moyamoya disease. It comprises arterial, capillary, venous, parenchymal, and pial compartments. Each component vasodilates by variable amounts following a drop in CPP. The capacity by which these vessels can dilate to maintain CBF during a fall in CPP is known as the CVR. Patients with moyamoya disease are often in a chronic state of cerebral vasodilation and therefore have a reduced or absent CVR.

Measuring CVR in patients with moyamoya disease is very informative, and this is often done by performing an Xe-CT or SPECT scan before and after administering a vasodilator, such as $\mathrm{CO}_{2}$ or acetazolamide (Diamox). More recently there has been interest in using ASL MR imaging for such challenge studies, given that it does not require contrast agents and therefore can be easily repeated within the same examination. The baseline and postvasodilator CBF values are then compared and CVR is calculated as the difference between the two. The CVR can therefore aid decision making for treatment options, 
Quantitative hemodynamic studies in moyamoya disease

TABLE 2: Main hemodynamic indicators of impending cerebral infarction in moyamoya disease based on imaging modality*

\begin{tabular}{|c|c|c|c|c|c|c|}
\hline PET & SPECT & Xe-CT & Perfusion CT & DSC & ASL & Doppler US \\
\hline$\downarrow \mathrm{CBF}$ & $\downarrow \mathrm{CVR} \dagger$ & $\downarrow \mathrm{CVR} \dagger$ & $\downarrow \mathrm{CBF}$ & $\uparrow \operatorname{Tmax}$ & $\downarrow \mathrm{CBF}$ & $\downarrow \mathrm{CBF}_{\ddagger}$ \\
\hline \multirow[t]{3}{*}{$\uparrow \mathrm{rOEF}$} & & & $\uparrow \mathrm{MTT}$ & $\uparrow \mathrm{MTT}$ & $\downarrow \mathrm{CBF} \dagger$ & \\
\hline & & & $\uparrow \mathrm{CBV}$ & $\begin{array}{l}\mathrm{PWl} / \mathrm{DWl} \\
\text { mismatch }\end{array}$ & & \\
\hline & & & $\downarrow \mathrm{CBF} \dagger$ & & & \\
\hline
\end{tabular}

* DWI $=$ diffusion-weighted imaging.

$\uparrow$ Postvasodilatory challenge (addition of acetazolamide [Diamox] or $\mathrm{CO}_{2}$ ).

$\ddagger$ Estimated and compared to contralateral hemisphere.

follow the natural course of the disease, and be used to evaluate the effects of surgery.

Table 2 summarizes the main hemodynamic findings that may indicate impending ischemia.

\section{Validity of Vasodilatory Tests}

Although vasodilatory tests such as the ones in which acetazolamide is administered appear useful in assessing CVR, there is still uncertainty about their accuracy and validity in guiding surgical treatments for moyamoya disease. Due to regional differences in vasodilatory capacity, there is in some instances a paradoxical decrease (rather than an increase or no change) in $\mathrm{rCBF}$ after acetazolamide administration. This so-called steal phenomenon is presumed to be caused by the redistribution of blood from regions in which vasodilation is at a maximum to areas where vasodilation can still occur.

\section{Cerebral Blood Flow and Age in Moyamoya Disease}

Kutsuzama and colleagues ${ }^{13}$ performed the first quantitative hemodynamic study in 6 patients with moyamoya disease in 1968, before the name of the disease was coined. Much that has been reported on quantitative hemodynamics in moyamoya disease relates to the pediatric population, and it is important to consider these results separately from those of adult patients with moyamoya. Normal CBF is known to correlate negatively with age. With the loss of neuronal mass, there is also a reduction in metabolic demand and therefore in the $\mathrm{CMRO}_{2}$. Furthermore, systemic factors in adults, such as atherosclerotic disease, diabetes, and hypertension may also play a role in affecting cerebral hemodynamics.

Several studies have examined the relationship between age and cerebral hemodynamics. There was general agreement that pediatric patients with moyamoya disease have a lower CBF compared with age-matched controls and adult patients with the disease, regardless of the method of analysis. ${ }^{6,11,12,14,24,29,36}$ The PET imaging studies also showed increased CBV and OEF more consistently in the pediatric population compared with adults. In addition, CVR, as determined by acetazolamide or $\mathrm{CO}_{2}$ challenge, was significantly impaired in the pediatric population. The pattern in adult patients was less clear-cut. This may explain why the most common clinical presentation of pediatric moyamoya disease is ischemic stroke and/or TIAs, whereas in adults hemorrhage appears more com- mon, at least in the Japanese population, in which most of these studies have been conducted. However, in some series, including our own experience of $>300$ patients treated at Stanford, the most common clinical presentation among adult patients was still ischemic stroke and/ or TIAs.

\section{Cerebral Blood Flow and Heterogeneity in Moyamoya Disease}

Some groups have also described a good correlation between the angiographic findings and the pattern of rCBF distribution..$^{9,23,25,33}$ Not surprisingly, because moyamoya disease predominantly affects the ICAs bilaterally, most often sparing the posterior circulation, the $\mathrm{rCBF}$ shows a loss of the normal pattern of frontal dominance. In its place, there is normal to increased $\mathrm{rCBF}$ in the occipital region.

In contrast to the pediatric population, there is less consensus regarding the hemodynamic findings in adult moyamoya disease. Some authors describe similar findings to those detailed above, ${ }^{15,25,34}$ whereas others have failed to show any significant difference between patients and age-matched controls. ${ }^{14,36}$ Perhaps adult patients with moyamoya disease constitute a more heterogeneous group, and selection bias may make comparing studies difficult. Piao and colleagues separated the adult patients who suffered a hemorrhage from those who suffered an ischemic stroke. The latter group was further separated angiographically into those with extensive basal moyamoya vessels and those without. These investigators demonstrated a significant difference in the hemodynamic status between the 2 groups, with a higher CBV and lower CVR in the group with extensive basal collateral vessels.

Similarly, segregation of patients into clinical subtypes has revealed markedly different hemodynamic results. Nariai et al. ${ }^{21}$ showed a significant increase in CBV and OEF in patients who had an infarction associated with TIAs, compared with patients who presented with either a hemorrhage or TIA. The latter 2 groups demonstrated no significant difference in their hemodynamic pattern compared with normal controls.

\section{Cerebral Blood Flow Postsurgery}

Quantitative hemodynamics enables better selection of patients for revascularization surgery and also postoperative evaluation of the clinical outcome. It also plays 
an important role in monitoring patients with or without treatment over time. Generally, patients who are asymptomatic but in whom moyamoya disease has been diagnosed angiographically should be monitored regularly. Only after a thorough quantitative hemodynamic evaluation should surgical treatment be considered if the patient becomes symptomatic.

Surgical revascularization is aimed at augmenting blood flow to the affected hemisphere, either directly with extracranial-intracranial bypass surgery, or indirectly via different techniques, including encephaloduroarteriosynangiosis, encephaloduroarteriomyosynangiosis, encephalomyosynangiosis, and placement of multiple bur holes. Most studies have documented clinical benefits and hemodynamic improvements after revascularization surgery. ${ }^{6,11,31,38}$ These studies demonstrate an improvement in the CVR and lowering of the CBV and OEF. Benefits may be seen as early as 3 months postrevascularization, although most follow-up studies were performed after 6 months.

A favorable clinical outcome is not necessarily matched with an improvement in the various hemodynamic parameters. ${ }^{29,30}$ The rCBF itself can often remain at preoperative levels despite improvements of other hemodynamic parameters. Some advocate that when postoperative evaluation demonstrates ongoing poor regional CVR, patients continue to be at risk for further ischemic injury. ${ }^{30}$ We suggest closer monitoring for this group of patients and consideration of further revascularization procedures if a patient continues to have worsening symptoms.

Intraoperative blood flow measurements in moyamoya disease can give valuable information from a single vessel distal to the stenoocclusion. Blood flow measurements immediately before and after microanastomosis allow a rapid assessment of the quality of the graft and the likely success of the anastomosis. ${ }^{1}$ We examined $>450$ revascularization procedures and could find no correlation between disease severity and blood flow measured at the site of microanastomosis. However, we detected a 4- to 5 -fold increase in the blood flow through the microanastomosis site after the procedure, and this correlated well with clinical improvement and increase in the CVR, as demonstrated using Xe-CT.

The details of this study will be discussed in depth elsewhere.

\section{Conclusions}

Quantitative hemodynamic techniques continue to improve with time, but with this improvement comes the difficulty in comparing studies in which different methodologies were used. However, most of the current techniques have been validated against each other. Patterns of hemodynamic compromise can be seen, especially in the pediatric group. This compromise consists of reduced $\mathrm{rCBF}$, increased regional $\mathrm{CBV}$ and $\mathrm{rOEF}$, and decreased CVR. Revascularization surgery usually brings about a clinical and hemodynamic improvement. The evaluation of adult moyamoya disease on the basis of clinical presentation and the presence or absence of extensive basal moyamoya vessels may help better elucidate the hemodynamic states, and it may also help guide patient selection for surgical treatment. Certainly, there are still controversies over the usefulness of various hemodynamic studies in the selection of patients for revascularization surgery. Further evaluation of each technique in patients with moyamoya disease is necessary.

\section{Disclaimer}

The authors report no conflict of interest concerning the materials or methods used in this study or the findings specified in this paper.

\section{References}

1. Amin-Hanjani S, Charbel FT: Flow-assisted surgical technique in cerebrovascular surgery. Surg Neurol 68 (1 Suppl): S4-S11, 2007

2. Derdeyn CP, Videen TO, Yundt KD, Fritsch SM, Carpenter DA, Grubb RL, et al: Variability of cerebral blood volume and oxygen extraction: stages of cerebral haemodynamic impairment revisited. Brain 125:595-607, 2002

3. Detre JA, Subramanian VH, Mitchell MD, Smith DS, Kobayashi A, Zaman A, et al: Measurement of regional cerebral blood flow in cat brain using intracarotid $2 \mathrm{H} 2 \mathrm{O}$ and $2 \mathrm{H} \mathrm{NMR}$ imaging. Magn Reson Med 14:389-395, 1990

4. Estol CJ: Dr C. Miller Fisher and the history of carotid artery disease. Stroke 27:559-566, 1996

5. Horowitz M, Yonas H, Albright AL: Evaluation of cerebral blood flow and hemodynamic reserve in symptomatic moyamoya disease using stable Xenon-CT blood flow. Surg Neurol 44:251-262, 1995

6. Ikezaki K, Matsushima T, Kuwabara Y, Suzuki SO, Nomura T, Fukui M: Cerebral circulation and oxygen metabolism in childhood moyamoya disease: a perioperative positron emission tomography study. J Neurosurg 81:843-850, 1994

7. Kang KH, Kim HS, Kim SY: Quantitative cerebrovascular reserve measured by acetazolamide-challenged dynamic CT perfusion in ischemic adult moyamoya disease: initial experience with angiographic correlation. AJNR Am J Neuroradiol 29:1487-1493, 2008

8. Kanno I, Uemura K, Higano S, Murakami M, Iida H, Miura $\mathrm{S}$, et al: Oxygen extraction fraction at maximally vasodilated tissue in the ischemic brain estimated from the regional $\mathrm{CO} 2$ responsiveness measured by positron emission tomography. $\mathbf{J}$ Cereb Blood Flow Metab 8:227-235, 1988

9. Karasawa J, Kikuchi H, Kuriyama Y, Sawada T, Kuro M, Kobayashi K, et al: [Cerebral hemodynamics in "moyamoya" disease-II. Measurements of cerebral circulation and metabolism by use of the argon desaturation method in pre- and postneurosurgical procedures (author's transl).] Neurol Med Chir (Tokyo) 21:1161-1168, 1981

10. Kety SS, Schmidt CF: The nitrous oxide method for the quantitative determination of cerebral blood flow in man; theory, procedure and normal values. J Clin Invest 27:476-483, 1948

11. Kuroda S, Houkin K, Kamiyama H, Abe H, Mitsumori K: Regional cerebral hemodynamics in childhood moyamoya disease. Childs Nerv Syst 11:584-590, 1995

12. Kuroda S, Kamiyama H, Abe H, Yamauchi T, Kohama Y, Houkin K, et al: Cerebral blood flow in children with spontaneous occlusion of the circle of Willis (moyamoya disease): comparison with healthy children and evaluation of annual changes. Neurol Med Chir (Tokyo) 33:434-438, 1993

13. Kutsuzama T, Takahashi S, Saito C, Sato T: Studies of cerebral hemodynamics in subarachnoid hemorrhage. Tohoku J Exp Med 94:407-415, 1968

14. Kuwabara Y, Ichiya Y, Otsuka M, Tahara T, Gunasekera R, 


\section{Quantitative hemodynamic studies in moyamoya disease}

Hasuo K, et al: Cerebral hemodynamic change in the child and the adult with moyamoya disease. Stroke 21:272-277, 1990

15. Kuwabara Y, Ichiya Y, Sasaki M, Yoshida T, Masuda K, Matsushima T, et al: Response to hypercapnia in moyamoya disease. Cerebrovascular response to hypercapnia in pediatric and adult patients with moyamoya disease. Stroke 28:701707,1997

16. Laborde G, Harders A, Klimek L, Hardenack M: Correlation between clinical, angiographic and transcranial Doppler sonographic findings in patients with moyamoya disease. Neurol Res 15:87-92, 1993

17. Lee YS, Jung KH, Roh JK: Diagnosis of moyamoya disease with transcranial Doppler sonography: correlation study with magnetic resonance angiography. J Neuroimaging 14:319323, 2004

18. McAuley DJ, Poskitt K, Steinbok P: Predicting stroke risk in pediatric moyamoya disease with xenon-enhanced computed tomography. Neurosurgery 55:327-333, 2004

19. Muttaqin Z, Ohba S, Arita K, Nakahara T, Pant B, Uozumi $\mathrm{T}$, et al: Cerebral circulation in moyamoya disease: a clinical study using transcranial Doppler sonography. Surg Neurol 40:306-313, 1993

20. Nambu K, Suzuki R, Hirakawa K: Cerebral blood flow: measurement with xenon-enhanced dynamic helical CT. Radiology 195:53-57, 1995

21. Nariai T, Matsushima Y, Imae S, Tanaka Y, Ishii K, Senda $\mathrm{M}$, et al: Severe haemodynamic stress in selected subtypes of patients with moyamoya disease: a positron emission tomography study. J Neurol Neurosurg Psychiatry 76:663-669, 2005

22. Nariai T, Senda M, Ishii K, Wakabayashi S, Yokota T, Toyama $\mathrm{H}$, et al: Posthyperventilatory steal response in chronic cerebral hemodynamic stress: a positron emission tomography study. Stroke 29:1281-1292, 1998

23. Ogawa A, Yoshimoto T, Suzuki J, Sakurai Y: Cerebral blood flow in moyamoya disease. Part 1: correlation with age and regional distribution. Acta Neurochir (Wien) 105:30-34, 1990

24. Østergaard L: Principles of cerebral perfusion by bolus tracking. J Magn Reson Imaging 22:710-717,1005

25. Piao R, Oku N, Kitagawa K, Imaizumi M, Matsushita K, Yoshikawa $\mathrm{T}$, et al: Cerebral hemodynamics and metabolism in adult moyamoya disease: comparison of angiographic collateral circulation. Ann Nucl Med 18:115-121, 2004

26. Rim NJ, Kim HS, Shin YS, Kim SY: Which CT perfusion parameter best reflects cerebrovascular reserve?: correlation of acetazolamide-challenged CT perfusion with single-photon emission CT in moyamoya patients. AJNR Am J Neuroradiol 29:1658-1663, 2008

27. Saito N, Nakagawara J, Nakamura H, Teramoto A: Assessment of cerebral hemodynamics in childhood moyamoya disease using a quantitative and a semiquantitative IMP-SPECT study. Ann Nucl Med 18:323-331, 2004

28. Sakamoto S, Ohba S, Shibukawa M, Kiura Y, Arita K, Kurisu $\mathrm{K}$ : CT perfusion imaging for childhood moyamoya disease before and after surgical revascularization. Acta Neurochir (Wien) 148:77-81, 2006

29. Sato H, Sato N, Tamaki N, Matsumoto S: Chronic low-perfusion state in children with moyamoya disease following revascularization. Childs Nerv Syst 6:166-171, 1990

30. So Y, Lee HY, Kim SK, Lee JS, Wang KC, Cho BK, et al: Prediction of the clinical outcome of pediatric moyamoya disease with postoperative basal/acetazolamide stress brain perfusion SPECT after revascularization surgery. Stroke 36:1485-1489, 2005
31. Suzuki J, Takaku A: Cerebrovascular "moyamoya" disease. Disease showing abnormal net-like vessels in base of brain. Arch Neurol 20:288-299, 1969

32. Suzuki R, Nariai T, Matsushima Y, Hirakawa K: Xe-CT in cerebrovascular disease and moyamoya disease. Acta Neurol Scand Suppl 166:69-71, 1996

33. Suzuki R, Tsuruoka S, Hiratsuka H, Matsushima Y, Fukumoto $\mathrm{T}$, Inaba $\mathrm{Y}$, et al: [Cerebral circulation in pediatric patients with moyamoya disease. Tomographic cerebral blood flow map obtained by xenon-enhanced computerized tomography.] Neurol Med Chir (Tokyo) 25:969-974, 1985 (Jpn)

34. Takeuchi S, Tanaka R, Ishii R, Tsuchida T, Kobayashi K, Arai $\mathrm{H}$ : Cerebral hemodynamics in patients with moyamoya disease. A study of regional cerebral blood flow by the $133 \mathrm{Xe}$ inhalation method. Surg Neurol 23:468-474, 1985

35. Taki W, Yonekawa Y, Kobayashi A, Ishikawa M, Kikuchi $\mathrm{H}$, Nishizawa S, et al: Cerebral circulation and oxygen metabolism in moyamoya disease of ischemic type in children. Childs Nerv Syst 4:259-262, 1988

36. Taki W, Yonekawa Y, Kobayashi A, Ishikawa M, Kikuchi $\mathrm{H}$, Nishizawa S, et al: Cerebral circulation and metabolism in adults' moyamoya disease-PET study. Acta Neurochir (Wien) 100:150-154, 1989

37. Tanaka Y, Nariai T, Nagaoka T, Akimoto H, Ishiwata K, Ishii $\mathrm{K}$, et al: Quantitative evaluation of cerebral hemodynamics in patients with moyamoya disease by dynamic susceptibility contrast magnetic resonance imaging-comparison with positron emission tomography. J Cereb Blood Flow Metab 26:291-300, 2006

38. Touho H, Karasawa J, Ohnishi H: Preoperative and postoperative evaluation of cerebral perfusion and vasodilatory capacity with 99mTc-HMPAO SPECT and acetazolamide in childhood Moyamoya disease. Stroke 27:282-289, 1996

39. Wintermark M, Maeder P, Verdun FR, Thiran JP, Valley JF, Schnyder P, et al: Using $80 \mathrm{kVp}$ versus $120 \mathrm{kVp}$ in perfusion CT measurement of regional cerebral blood flow. AJNR Am J Neuroradiol 21:1881-1884, 2000

40. Wintermark M, Sesay M, Barbier E, Borbely K, Dillon WP, Eastwood JD, et al: Comparative overview of brain perfusion imaging techniques. Stroke 36:e83-e99, 2005

41. Zaharchuk G, Mandeville JB, Bogdanov AA Jr, Weissleder R, Rosen BR, Marota JJ: Cerebrovascular dynamics of autoregulation and hypoperfusion. An MRI study of CBF and changes in total and microvascular cerebral blood volume during hemorrhagic hypotension. Stroke 30:2197-2205, 1999

Manuscript submitted December 14, 2008.

Accepted January 26, 2009.

This work was supported in part by Russell and Elizabeth Siegelman, Bernard and Ronni Lacroute, and the William Randolph Hearst Foundation.

Address correspondence to: Marco Lee, M.D., Ph.D., Department of Neurosurgery, Stanford University Medical Center, 300 Pasteur Drive, Stanford, California 94305. email: marcolee@stanford.edu. 